\title{
Argentina-Alzheimer's disease neuroimaging initiative (Arg-ADNI): neuropsychological evolution profile after one-year follow up
}

\author{
Argentina-Alzheimer's disease neuroimaging initiative (Arg-ADNI): evolução do perfil \\ neuropsicológico depois de um ano de seguimento \\ Patricio Chrem Méndez', Ismael Calandri', Federico Nahas', María Julieta Russo', Ignacio Demey', \\ María Eugenia Martín', María Florencia Clarens ${ }^{1}$, Paula Harris', Fernanda Tapajoz', Jorge Campos', \\ Ezequiel I. Surace1, Horacio Martinetto', Fernando Ventrice1, Gabriela Cohen', Silvia Vázquez', \\ Carlos Romero', Salvador Guinjoan ${ }^{1}$, Ricardo F. Allegri', Gustavo Sevlever ${ }^{1}$
}

\begin{abstract}
The Argentina-Alzheimer's disease neuroimaging initiative (Arg-ADNI) study is a longitudinal prospective cohort of 50 participants at a single institution in Buenos Aires, Argentina. Longitudinal assessments on a neuropsychological test battery were performed on 15 controls, 24 mild cognitive impairment (MCl) patients and 12 Alzheimer's disease (AD) dementia patients. In our study population, there was a high prevalence of positive AD biomarkers in the AD group, 92.3\% (12/13); and a low prevalence in the normal controls, 20\%; almost half (48\%) of the patients diagnosed with $\mathrm{MCl}$ had positive amyloid detection. After a one year, the significant differences found at baseline on neuropsychological testing were similar at the follow-up assessment even though the AD group had significantly altered its functional performance (FAQ and CDR). The exception was semantic fluency, which showed greater impairment between the $A D$ group and $M C I$ and normal controls respectively. For these tests, the addition of AD biomarkers as a variable did not significantly alter the variations previously found for the established clinical group's model. Finally, the one-year conversion rate to dementia was $20 \%$ in the $\mathrm{MCl}$ cohort.
\end{abstract}

Keywords: Alzheimer's disease; cognitive dysfunction; biomarkers; neuroimaging.

\section{RESUMO}

El estudio de Argentina-Alzheimer's Disease Neuroimaging Initiative (Arg-ADNI) es una cohorte prospectiva de 50 pacientes seguidos en una misma institución. Fueron evaluados cognitivamente 15 controles normales (CN), 24 sujetos con deterioro cognitivo leve (DCL) y 12 con demencia tipo Alzheimer (DTA) leve. En los DTA, 92,3\% tuvieron biomarcadores positivos para Alzheimer y 20\% en los CN. Casi la mitad de los DCL presentaron biomarcadores positivos. Después de un año de seguimiento, la diferencias significativas halladas en la visita de inicio en las pruebas cognitivas fueron similares al año aunque los DTA tuvieron empeoramiento funcional medido en el FAQ y CDR. La excepción fue la fluencia semántica, la cual mostró mayor declinación entre DTA y los demás grupos. La incorporación de los biomarcadores como variable no alteró significativamente los hallazgos de grupo. La tasa de conversión a demencia anual fue del 20\%.

Palabras-clave: enfermedad de Alzheimer; disfunción cognitiva; biomarcadores; neuroimagen.

Alzheimer's disease $(\mathrm{AD})$ is the most common neurodegenerative disease causing dementia. Although there are many genetic and environmental risk factors, age is the most important risk factor that leads to sporadic $\mathrm{AD}$. As the world population ages, $\mathrm{AD}$ is becoming more prevalent ${ }^{1,2}$. Moreover, aging of the population will be more pronounced in underdeveloped countries ${ }^{3}$, making South America a vulnerable region ${ }^{4}$. Despite these predictions, few governmental policies have been made in Latin America to tackle this epidemiological problem in a comprehensive way.

The Alzheimer's Disease Neuroimaging Initiative (ADNI) is a worldwide, standardized, observational, and multicenter study that analyzes longitudinal changes in clinical, imaging, genetic, and biochemical biomarkers for the early

Fundación para la Lucha contra las Enfermedades Neurologicas de la Infancia, Instituto de Investigaciones Neurológicas Raúl Carrea, Aging and Memory Center, Buenos Aires, Argentina.

Correspondence: Patricio Chrem Mendez; Memory and Aging Center, Institute FLENI, Montañeses 2325 C1428AQK, Buenos Aires, Argentina; E-mail: pchremmendez@fleni.org.ar

Conflict of interest: There is no conflict of interest to declare.

Support: FLENI Foundation supported this work.

Received 19 September 2017; Received in final form 08 January 2018; Accepted 08 January 2018. 
detection and tracking of $\mathrm{AD}$ in participants who meet the criteria for mild cognitive impairment (MCI) and mild AD, including elderly normal controls. The aim of the ADNI is to define the progression of $\mathrm{AD}$, as well as to develop reliable biomarkers as predictors of this disease. The study began in 2004 and has been extended three times since its inception $^{5}$. The Argentina ADNI (Arg-ADNI) has launched the first South American ADNI effort to acquire data in a collaborative study ${ }^{6}$. Although our center committed to assess a small cohort, this represented the first South American population that has been studied in an exhaustive and standardized manner.

The aim of this article is to show data on longitudinal changes in neuropsychological and biomarker testing after one year follow-up in the three clinical, well-studied groups of the first South American ADNI population.

\section{METHODS}

This was a one-year observational and longitudinal study, which comprises the results of neuropsychological testing in a well-studied population that is part of the ongoing ArgADNI. In agreement with other worldwide ADNI programs, Arg-ADNI follows the same study design to guarantee a standard and uniform evaluation of participants ${ }^{6}$. A cohort of 56 individuals between the ages of 55 and 85 were enrolled at the Neurological Research Institute, Foundation for the Fight Against Neurological Diseases of Children / Fundación para la Lucha contra las Enfermedades Neurológicas de la Infancia (FLENI) and were divided into three possible clinical groups according to the inclusion and exclusion criteria and sample classification: 15 cognitively normal participants were included in the normal control group (NC group), 27 patients in the mild cognitive impairment group (MCI group) and 14 patients diagnosed with Alzheimer's disease (AD group). Each participant from all three groups underwent a baseline assessment, in which a structured evaluation was performed including: clinical and neurological examination, neuropsychological test battery, genetic blood test assay, cerebrospinal fluid (CSF) AD biomarkers, brain 3.0 T MRI scan, FDGPET scan and PiB-PET scan; and then a follow-up visit that was performed after 12 months after the first evaluation.

\section{Standard protocol approvals and patient consent}

This study received the approval of the FLENI bioethics board. Each participant, with their corresponding study partner, signed the informed consent form before initial evaluation.

\section{Inclusion and exclusion criteria}

The inclusion criteria were as follows:

1) Participants with a Hachinski Ischemic Scale ${ }^{7}$ score $\leq 4$; the Geriatric Depression Scale ${ }^{8}$ score $<6$ and an education of at least grade 6 or work history equivalent.
2) A study partner with 10 hours per week of contact, either in person or on the telephone, who could accompany the participant to the clinical visits.

3) A list of allowed medications that were stable for four weeks prior to the baseline evaluation.

4) Visual and auditory acuity adequate for neuropsychological testing.

5) Exclusion criteria were:

6) Major depression, bipolar disorder as described in the DSM-IV within the past year.

7) Psychotic features, agitation or behavioral problems within the last three months that could lead to difficulty complying with the protocol.

8) History of schizophrenia (DSM-IV criteria).

9) History of alcohol or substance abuse or dependence within the past two years (DSM-IV criteria).

10) Any significant systemic illness or unstable medical condition that could lead to difficulty complying with the protocol.

11) Women had to be sterile or two years past childbearing potential.

12) Participants could not have any medical contraindication to MRI or PET and could not be enrolled concurrently in other trials or studies.

\section{Sample classification}

The classification criteria for each of the groups were as follows: In regard to memory complaints, participants in the NC group had to be free of any complaint, while the MCI and AD patients both had to have reported memory impairment. On the Mini-Mental State Examination (MMSE) ${ }^{9,10}$, the range for normal participants and those with MCI was 24-30, and for AD was 20-26; all inclusive. The Clinical Dementia Rating (CDR $)^{11}$ score for normal participants was 0 and for participants with MCI was 0.5 with a mandatory requirement of the memory box score being $\geq 0.5$, and the rating for patients with $\mathrm{AD}$ was 0.5 or 1 . For the memory criterion, delayed recall of paragraph 1 from the Logical Memory II subscale of the Wechsler Memory Scale-Revised ${ }^{12}$ (maximum score of 25) was used, with cutoff scores as follows based on education: for normal participants we established a cutoff score $\geq 9$ for 16 years of education, between 5-8 for 8-15 years of education, and between 3-7 for 0-7 years of education. For participants with MCI and those with $\mathrm{AD}$, the cutoff scores were $\leq 8$ for 16 years of education, $\leq 4$ for 8-15 years of education, and $\leq 2$ for $0-7$ years of education. In addition, the $\mathrm{NC}$ participants had to be age-matched to the other participants and could not have any significant impairment in cognitive functions or activities of daily living. The participants with MCI had to be intact with regard to functional performance, and could not qualify for the diagnosis of dementia. The participants with $\mathrm{AD}$ had to be at the mild stage of the disease and had to meet the National Institute of Neurological and Communicative Disorders and Stroke-Alzheimer's disease and Related Disorders Association criteria for probable $\mathrm{AD}^{13}$, regardless whether the CDR had been scored as 0.5 or 1 . 


\section{Baseline visit}

At the baseline visit, each participant provided all their data about demographics, family and medical history. In addition, they were given a clinical and neurological examination, neuropsychological test battery, CSF AD biomarker measure, brain 3T MRI scan, FDG-PET scan and PiB-PET scan. It usually took two or three visits to our center for participants to complete the baseline visit, although the timeframe for its completion was never over a month.

\section{Neuropsychological test battery and MRI scan}

At baseline, as well as at the one-year follow-up, participants were given the Alzheimer's Disease Assessment Scale $(\mathrm{ADAS})^{14,15}$, Montreal Cognitive Assessment (MoCA) $)^{16,17}$, Clock Drawing Test ${ }^{18}$, Rey Auditory Verbal Learning Test ${ }^{19,20}$ (RAVLT: total, delayed recall, recognition score), Boston Naming Test ${ }^{21}$, Semantic Fluency, Phonological Fluency ${ }^{22}$, Trail Making Test A and B ${ }^{23,24}$ (TMT-A, TMT-B), Geriatric Depression Scale ${ }^{8}$, and Functional Assessment Questionnaire (FAQ) ${ }^{25}$.

All participants also underwent a brain MRI with a 3 Tesla strength scanner including T1, T2, FLAIR, GRE and DWI sequences. Images were saved in a Kodak Carestream System at FLENI for clinical reviewing. No participant was excluded for having MRI findings consistent with other brain pathologies that could be the cause of cognitive impairment. All the MRI images were examined by a radiologist as well as retrospectively by the review board of the Arg-ADNI.

\section{${ }^{11} \mathrm{C}$-PiB-PET synthesis and purification}

Synthesis of ${ }^{11} \mathrm{C}-\mathrm{PiB}$ was performed in the TRACERlab FXC Pro Chemistry Synthesizer. The duration of the whole process, from receipt of ${ }^{11} \mathrm{C}-\mathrm{CO}_{2}$ to subsequent purification was 35 minutes. Purification was performed through the differential interaction among the components of the raw product, the stationary and the mobile phases. The final purification was performed within the synthesis module. The final result was a mixed solution of the mobile phase with a volume of $28 \mathrm{ml}$ physiological solution with a content of less than $10 \%$ ethanol and approximately $200 \mathrm{mCi}{ }^{11} \mathrm{C}-\mathrm{PiB}$. The final result of the reaction was $10 \%$.

After 50 minutes of the endovenous infusion of $10 \mathrm{mCi}$ of ${ }^{11} \mathrm{C}-\mathrm{PiB}$, volumetric multislice computerized tomography (CT) brain images were obtained using PET/CT Discovery 690 GE equipment. Images were obtained using a nonuniform attenuation correction with CT. Axial, coronal and sagittal images were obtained either with MRI and/or CT.

\section{PET visual analysis}

Images were viewed and analyzed by two nuclear medicine physicians, blinded to the clinical data of the patients. The presence or absence of cortical amyloid and its spatial distribution was analyzed qualitatively using visual color linear scales. Based on the concentration of activity of ${ }^{11} \mathrm{C}-\mathrm{PiB}$, the degree of cortical retention was classified as positive or negative. A total of 50 out of 56 patients underwent a PiB-PET $\operatorname{scan}(89.2 \%)$.

\section{CSFAD biomarkers}

The CSF was obtained by lumbar puncture from 40 out of 56 participants (71.4\%). Core AD biomarkers $A \beta 1-42$, total tau and phosphorylated tau at T181 were quantified using the INNOTEST' (Fujirebio) enzyme-linked immunosorbent assay kit. Cutoff values considered by our local laboratory as a typical $\mathrm{AD}$ biomarker signature are: $<532 \mathrm{pg} / \mathrm{ml}$ for $\mathrm{A} \beta$, > $100 \mathrm{pg} / \mathrm{dl}$ for total tau protein and $>26.5 \mathrm{pg} / \mathrm{ml}$ for tau phosphorylated ${ }^{26}$. All three measures had to be in the range for $\mathrm{AD}$ to consider the individual as positive.

\section{$A D$ biomarker considerations}

In our cohort, not every participant underwent both lumbar puncture and a PiB-PET scan and there were three patients (two $\mathrm{MCI}$ and one $\mathrm{AD}$ ) who, although they were included at the baseline analysis, refused both imaging and biochemical biomarker analysis. However, these participants were excluded during the follow-up for other reasons as well (Figure 1). For the remaining 53 participants, at least one or the other of these two biomarkers were analyzed. For the purposes of analysis and diagnostic certainty, we considered a subgroup of participants to be "positive" for $\mathrm{AD}$ biomarkers when at least one of these assays was positive on amyloid detection and a "negative $\mathrm{AD}$ biomarker" subgroup when at least one of the assays were negative. No discrepancies were found in those participants who had both $\mathrm{AD}$ biomarkers performed.

\section{One-year follow-up visit}

From the initial cohort of 56 participants, 50 were followed up and completed the one-year follow-up visit. In this visit, participants underwent clinical and neurological evaluation. The same neuropsychological test battery as for the baseline visit was used, with the exception of the RAVLT, which included a different list. Also, a new MRI scan was performed. Conversion to dementia was measured by the CDR score.

\section{Data analysis}

All data analysis was done using IBM SPSS software. For baseline analysis, we used parametric (one way-ANOVA) and non-parametric (Pearson's Chi-square) tests when necessary. Post hoc group and magnitude effect analyses were performed using Bonferroni's method. For all neuropsychological variables, we performed a baseline group analysis and a one-year follow-up analysis using ANOVA to assess differences between the clinical groups. To address the impact of time and the biomarker status in the patients' evolution, we performed a repeated measures ANOVA with clinical groups and biomarker status as factors. To address the assumptions needed in this model, 
we performed the Levene, Box Cox and Mauchly diagnostic tests. A Huynh-Feldt correction was used to measure intra-participant variations p-value. Standardized scores (z-scores) were used when analyzing neuropsychological tests when applicable. For standardization, we used the mean and standard deviation (SD) from the FLENI-Aging and Memory Center normal controls' database.

Table 1. Population features at baseline visit.

\begin{tabular}{|c|c|c|c|c|}
\hline Variable & NC & $\mathrm{MCl}$ & $A D$ & $p$-value \\
\hline$n$ & 15 & 27 & 14 & \\
\hline Age* & $\begin{array}{l}66.7 \\
(7.02)\end{array}$ & $\begin{array}{l}73.12 \\
(5,16)\end{array}$ & 75.25 & $\begin{array}{c}0.018 \\
\text { (ANOVA) }\end{array}$ \\
\hline Sex (male) & 5 & 12 & 7 & $\begin{array}{c}0.642 \\
\text { (chi square) }\end{array}$ \\
\hline Education (years) & $\begin{array}{l}15.3 \\
(3.37)\end{array}$ & $\begin{array}{l}11.75 \\
(3.88)\end{array}$ & $\begin{array}{l}11.75 \\
(3.99)\end{array}$ & $\begin{array}{c}0.356 \\
\text { (ANOVA) }\end{array}$ \\
\hline CSF biomarkers + & $\begin{array}{c}2 / 11 \\
(18.2 \%)\end{array}$ & $\begin{array}{l}10 / 20 \\
(50 \%)\end{array}$ & $\begin{array}{c}8 / 9 \\
(88.9 \%)\end{array}$ & 0.07 \\
\hline $\mathrm{PiB}+$ & $\begin{array}{c}2 / 15 \\
(13.3 \%)\end{array}$ & $\begin{array}{c}10 / 22 \\
(45.5 \%)\end{array}$ & $\begin{array}{c}11 / 13 \\
(84.6 \%)\end{array}$ & $\begin{array}{c}0.01 \\
\text { (chi square) }\end{array}$ \\
\hline $\begin{array}{l}\text { Biomarker status } \\
\text { (positive) }\end{array}$ & $\begin{array}{l}3 / 15 \\
(20 \%)\end{array}$ & $\begin{array}{l}12 / 25 \\
(48 \%)\end{array}$ & $\begin{array}{c}12 / 13 \\
(92.3 \%)\end{array}$ & 0.000 \\
\hline
\end{tabular}

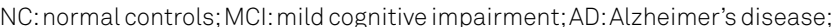
CSF: cerebrospinal fluid biomarkers; PiB: Pittsburgh compound B; +: positive.

\section{RESULTS}

\section{Baseline characteristics of study participants}

Information on the demographic characteristics of the groups are summarized in Table 1 . A total of 56 participants were included at the baseline evaluation. Notably, participants in the $\mathrm{AD}$ group were significantly older than those in the NC group, with a mean difference $-7 \pm 2.5(\mathrm{p}=0.018)$. No significant differences were observed between the $\mathrm{AD}$ and MCI groups. All three groups were similar with respect to years of education and gender. All participants were of European descent and Spanish-speaking. Fifty-three of the 56 participants had at least one AD biomarker performed (CSF 71.4\%; PiB-PET 89.2\%). The distribution of positive AD biomarkers between groups was similar for both methods (CSF and PiB-PET), with significant differences in the frequency of positivity of the NC group compared with the AD group (CSF $p=0.07$; PiB-PET $p=0.01$ ). In the case of the MCI group, there was a finding of positive amyloid detection in almost half of the participants (CSF 50\%; PiB 45.5\%).

Table 2 shows the mean scores and SD of the neuropsychological testing between groups at the baseline visit. The three groups significantly differed from each other in the following tests: the RAVLT immediate recall, delayed

Table 2. Neuropsychological features at baseline visit.

\begin{tabular}{|c|c|c|c|c|c|c|c|c|}
\hline \multirow{2}{*}{ Variable } & \multicolumn{2}{|c|}{ NC } & \multicolumn{2}{|c|}{$\mathrm{MCl}$} & \multicolumn{2}{|c|}{$A D$} & \multirow{2}{*}{ Significance } & \multirow{2}{*}{$p$-value } \\
\hline & Mean & SD & Mean & SD & Mean & SD & & \\
\hline$M_{M S E}^{b, c}$ & 29.21 & 1.53 & 28.63 & 1.15 & 21.64 & 3.5 & $\star$ & 0.000 \\
\hline Logical - immediate ${ }^{a, b, c}$ & 10.2 & 2.57 & 6.5 & 3.10 & 2.14 & 1.83 & * & 0.000 \\
\hline Logical - delayed ${ }^{a, b, c}$ & 7,93 & 2.15 & 3.92 & 3.45 & 0.5 & 1.09 & $\star$ & 0.000 \\
\hline CDR - Totalala,b,c & 0.03 & 0.13 & 0.5 & 0 & 0.78 & 0.42 & * & 0.000 \\
\hline ADAS $-11^{b, c}$ & 6.64 & 3.78 & 8.48 & 3.23 & 19.30 & 6.39 & $\star$ & 0.000 \\
\hline ADAS - $13^{b . c}$ & 11.53 & 4.63 & 15.73 & 3.65 & 30.07 & 8.28 & $\star$ & 0.000 \\
\hline Rey - immediate ${ }^{a, b, c}$ & 42.86 & 8.34 & 30.80 & 8.49 & 18.86 & 7.87 & $\star$ & 0.000 \\
\hline Rey - delayed a,b,c & 8.2 & 2.39 & 3.1 & 2.5 & 0.57 & 1.01 & * & 0.000 \\
\hline Rey - delayed - recognition ${ }^{a, b, c}$ & 12.73 & 1.79 & 9.96 & 3.39 & 6.08 & 3.20 & * & 0.000 \\
\hline MoCA ${ }^{a, b, c}$ & 27,77 & 2.20 & 23.04 & 3.51 & 16.36 & 4.08 & * & 0.000 \\
\hline Fluency - $\mathrm{P}^{\mathrm{b}}$ & 19.46 & 4.29 & 15.48 & 5.19 & 14.07 & 5.19 & * & 0.013 \\
\hline Fluency - Sem ${ }^{a, b, c}$ & 21.46 & 3.35 & 17.84 & 3.03 & 12.07 & 3.81 & * & 0.000 \\
\hline Clock Design b,c & 5 & 0 & 4.33 & 1.00 & 2.93 & 1.64 & * & 0.000 \\
\hline Clock - Copy ${ }^{b, c}$ & 5 & 0 & 4.96 & 0.20 & 4.43 & 1.16 & * & 0.021 \\
\hline TMT-A ${ }^{b, c}$ & 33.8 & 9.67 & 51.11 & 21.41 & 105.78 & 80.94 & * & 0.000 \\
\hline TMT-B $\mathrm{B}^{\mathrm{a}, \mathrm{b}, \mathrm{c}}$ & 75.8 & 29.05 & 143.33 & 78.62 & 244.71 & 83.46 & * & 0.000 \\
\hline$F A Q^{b, c}$ & 0 & 0 & 2.4 & 3.45 & 12.7 & 9.3 & * & 0.000 \\
\hline GDS & 1.7 & 1.49 & 2.7 & 2.4 & 1.7 & 1.61 & NS & 0.295 \\
\hline Boston b,c & 28 & 2.39 & 26.41 & 2.98 & 20.57 & 5.73 & * & 0.000 \\
\hline
\end{tabular}

MMSE: Mini Mental State Examination; Logical memory: Logical Memory II subscale of the Wechsler Memory Scale-Revised (immediate and delayed recall); ADAS: Alzheimer's disease Assessment Scale; Rey: Rey auditory verbal learning test (immediate, delay and recognition); MoCA: Montreal Cognitive Assessment; Fluency - P: Phonological fluency; Fluency - Sem: Semantic fluency. Clock: Clock Drawing Test; TMT-A and TMT-B: Trail Making Test A and B; FAQ: Functional Assessment Questionnaire; GDS: Geriatric Depression Scale; Boston: Boston Naming Test. a. differences between control group and MCI group; b. differences between Normal Control group and Alzheimer's dementia; c. differences between MCl group and Alzheimer's dementia group. 
recall and recognition (all three phases of memory), MoCA test, Semantic Fluency and TMT-B $(p=0.000)$. For the baseline analysis, the MMSE, Logical Memory and CDR were not considered, as these tests were used as part of the sample classification process, but they were considered in subsequent longitudinal analysis. For the rest of the neuropsychological tests, we found significant differences between the NC and AD groups, as well as between the MCI and AD groups, but not between the NC and MCI groups. There was no significant difference in the Geriatric Depression Scale between any of the groups. The NC group had no functional impairment as shown in the FAQ; however, there was a significant difference between the $\mathrm{MCI}$ versus the $\mathrm{AD}$ group, as expected (MCI mean score 2.4 versus $\mathrm{AD}$ mean score $12.7 ; \mathrm{p}=0.000$ ).

\section{Longitudinal analysis}

As mentioned above, 50 participants completed the one-year follow-up visit, as six participants were excluded during the follow-up, causing slight changes in the total number of participants in each group: NC $(n=14)$; $\mathrm{MCI}(\mathrm{n}=24)$ and $\mathrm{AD}(\mathrm{n}=12)$ (Figure 1$)$. Regarding the clinical status, five MCI participants had converted to dementia (AD group) based on functional worsening measured by the CDR (conversion from CDR 0.5 to 1 ). This represented a rate of conversion of $20 \%$ at the one-year follow-up. Four of these participants had a positive $\mathrm{AD}$ biomarker (only one was negative). For the purpose of longitudinal analysis of the neuropsychological testing, these participants were kept in the MCI group preserving the initial classification (Figure 1).

Figure 2 shows the evolution of scores of the neuropsychological testing indicating the mean values and SD between baseline (T1) and annual visit (T2) for each clinical group. To achieve a better correlation between the test results and age and educational level, z scores were used for most of the tests, except for the MoCA, ADAS-cog, Logical Memory, FAQ and CDR, in which the sum of boxes was used instead. In addition, and next to the graphs of the clinical groups, the same model was drawn showing those participants who had positive $\mathrm{AD}$ biomarkers versus those who were negative.

The Logical Memory test was included in the longitudinal analysis. After one year, the significant differences between groups remained similar to the baseline visit $(\mathrm{p}=0.000)$ and time did not cause a significant decline in total scores for both immediate and delayed recall.

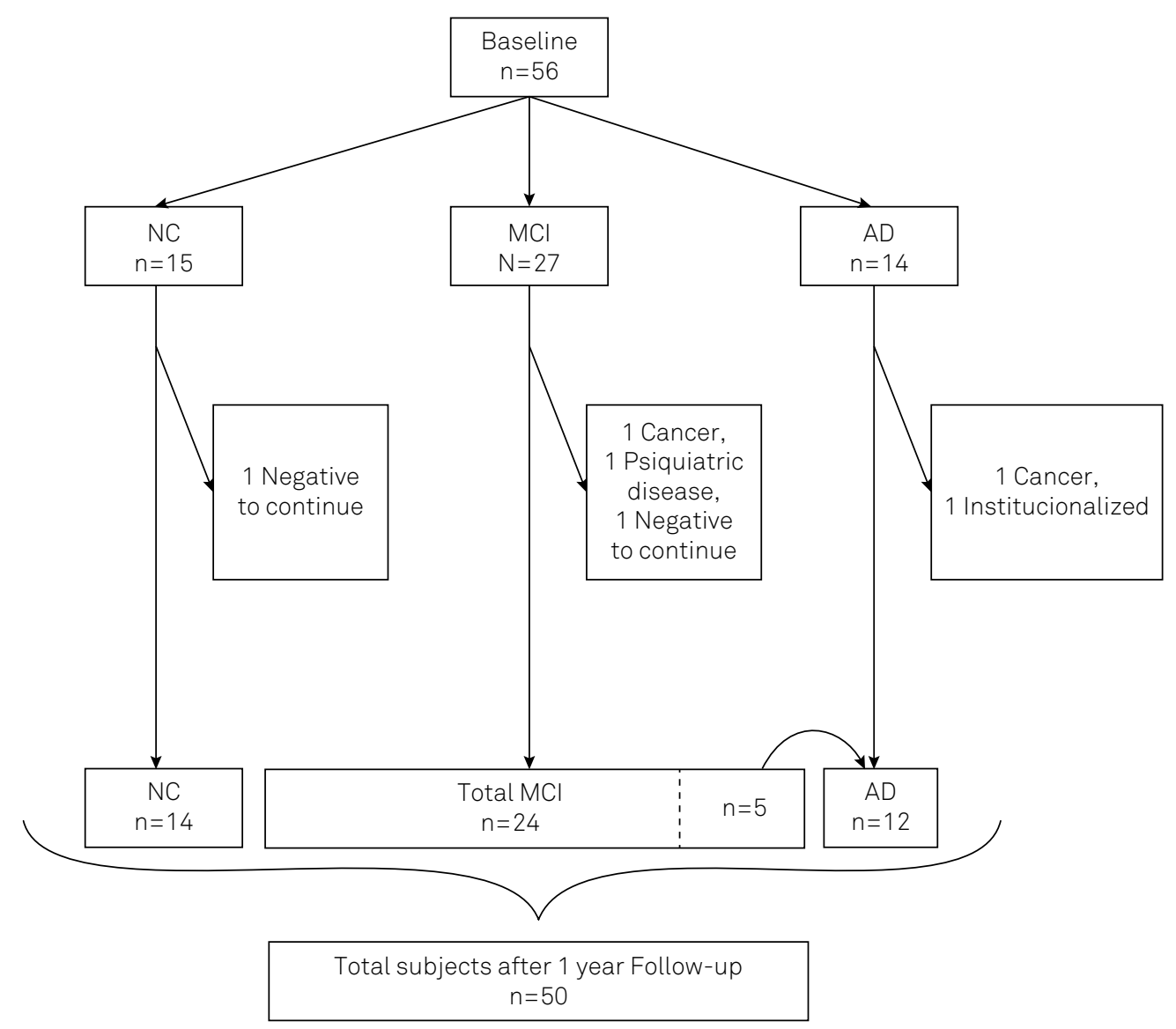

3rd group from top: Middle, line 2 should read: Psychiatric disease; Far right box line 2 should read: institutionalized; Bottom row should read: Total participants (NOT subjects)

Figure 1. Participants flowchart after one-year follow-up. 
The presence of $\mathrm{AD}$ biomarkers in this test did not change the evolution slopes significantly. However, we observed a trend towards improvement for those who were negative for $\mathrm{AD}$ biomarkers. This same situation was found for the
RAVLT, as significant differences were found after one year ( $p=0.000$ ) but there were no significant changes in the total scores. In this case, the presence of $\mathrm{AD}$ biomarkers did not modify the results slope.

\section{SHORT TEST}

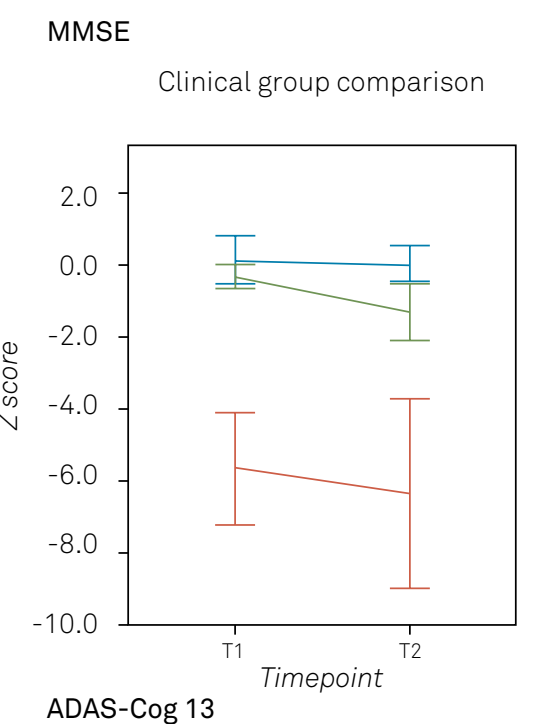

Clinical group comparison

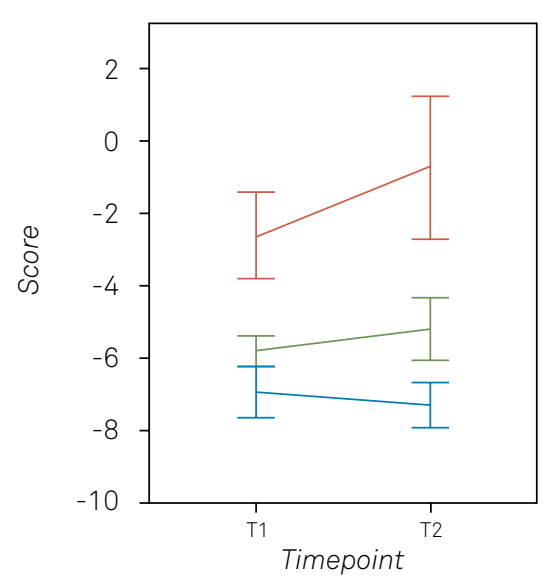

MoCA

Clinical group comparison

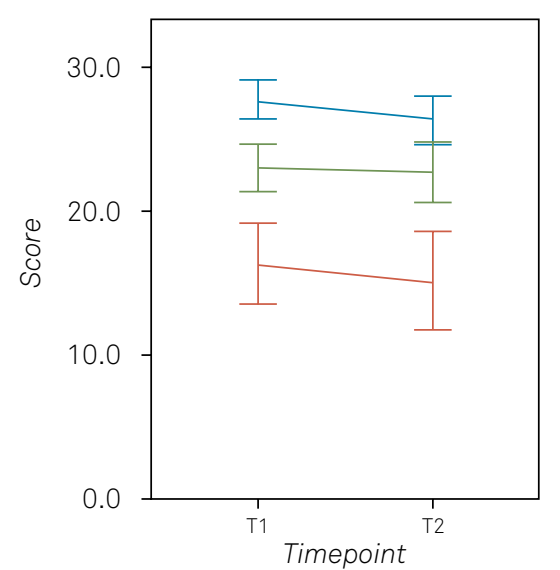

Clinical and biomarker group comparison

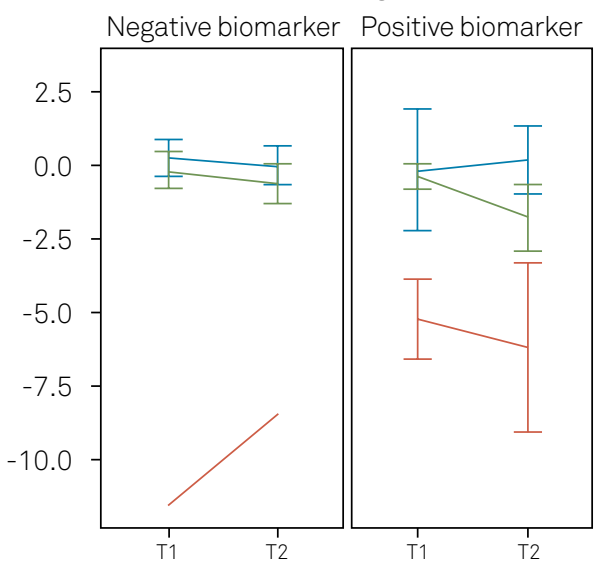

Clinical and biomarker group comparison Negative biomarker Positive biomarker

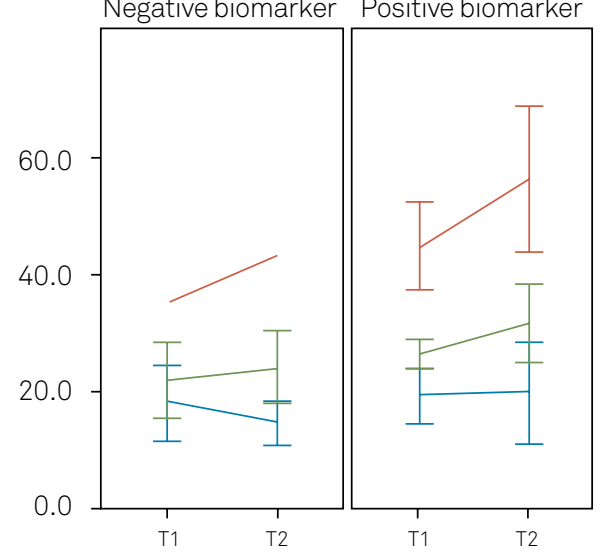

Clinical and Biomarker group comparison

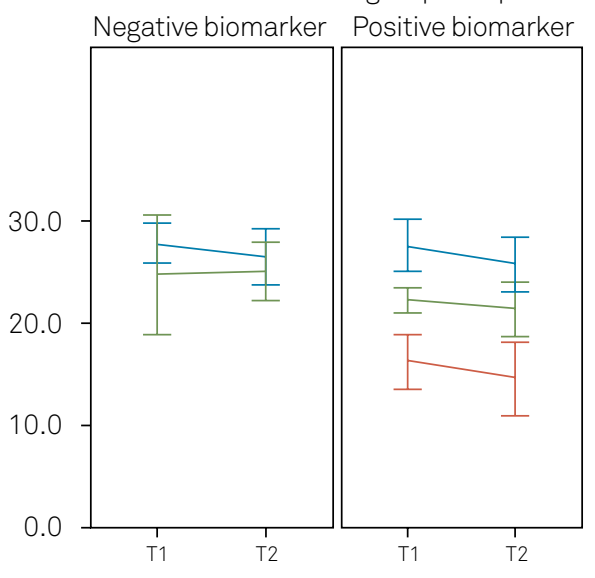

Green line: NC group; Yellow line: MCI group; Red line: AD group;T1: Baseline visit; T2: One-year follow-up.

Figure 2. Evolution line graphs for each clinical group and the respective neuropsychological test; and comparison of groups for the AD biomarker status (positive versus negative). 
Logical memory (I)

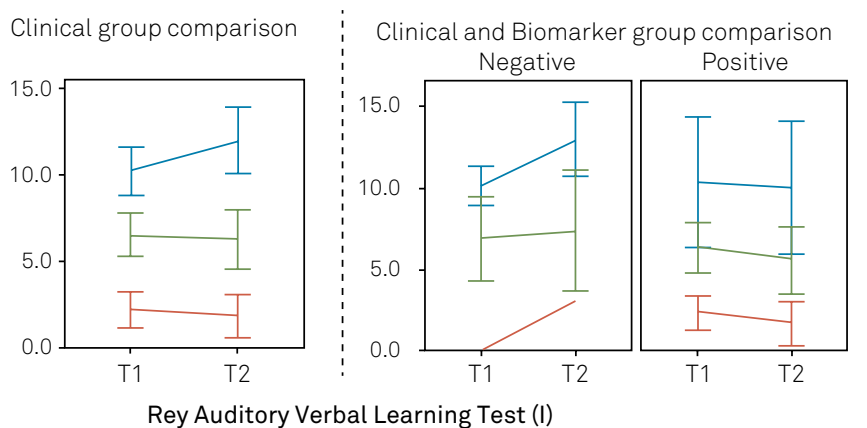

Clinical group comparison

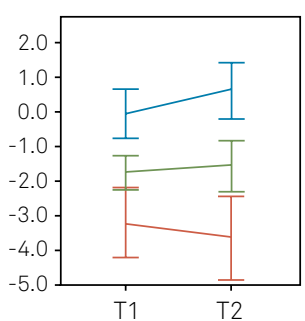

Clinical and Biomarker group comparison

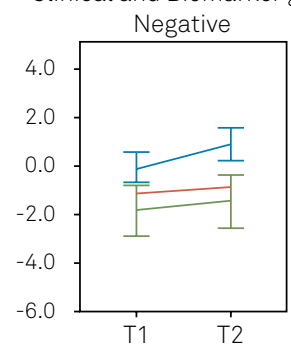
Positive

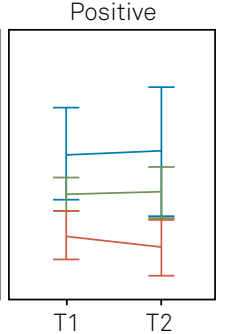

TMT A

Logical memory (D)

Clinical group comparison $\quad$ Clinical and Biomarker group comparison
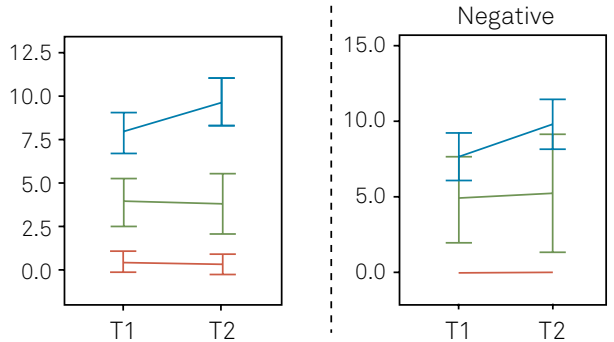

Positive

Rey Auditory Verbal Learning Test (delay)

Clinical group comparison $\quad$ Clinical and Biomarker group comparison
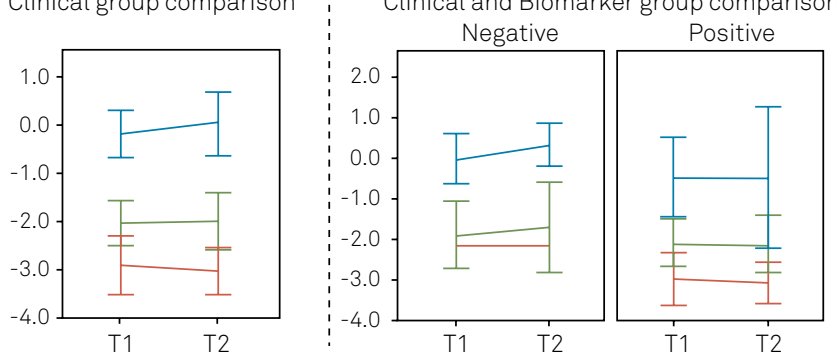

NON-MEMORY TEST

\section{TMT B}
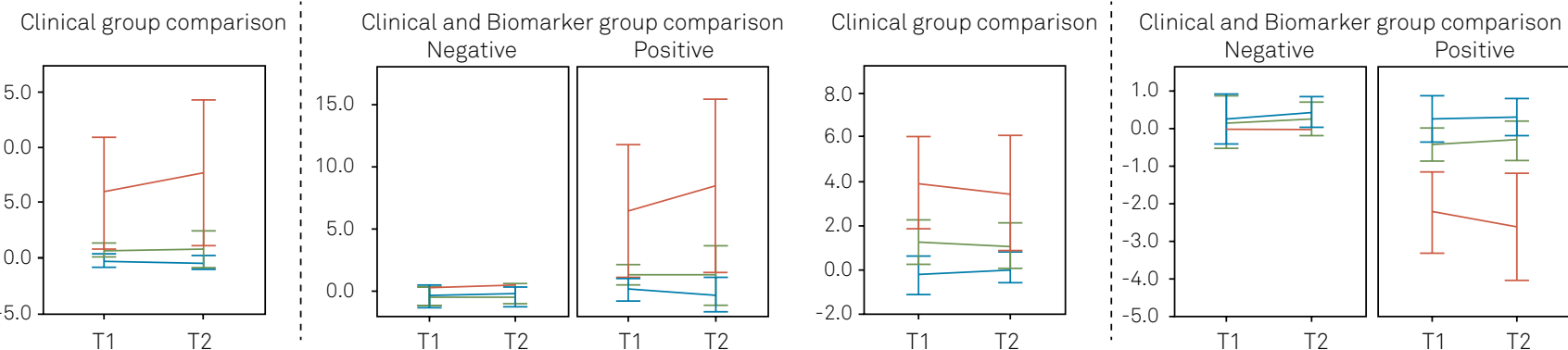

Phonological Fluency

LANGUAGE ASSESSMENT AND EVOLUTION

Semantic Fluency

Clinical group comparison

Clinical and Biomarker group comparison

Clinical group comparison

Clinical and Biomarker group comparison
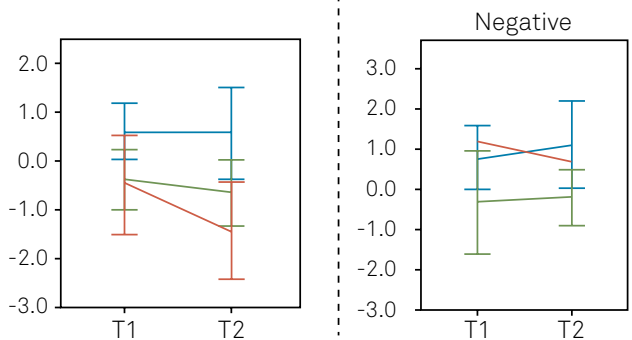
Positive
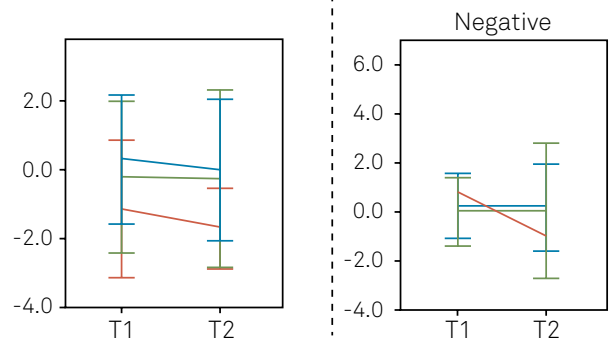
Positive

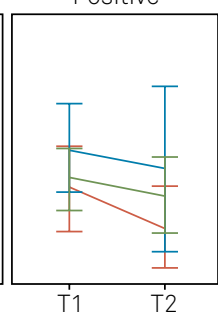

\section{Boston Naming Test}

Clinical group comparison i Clinical and Biomarker group comparison
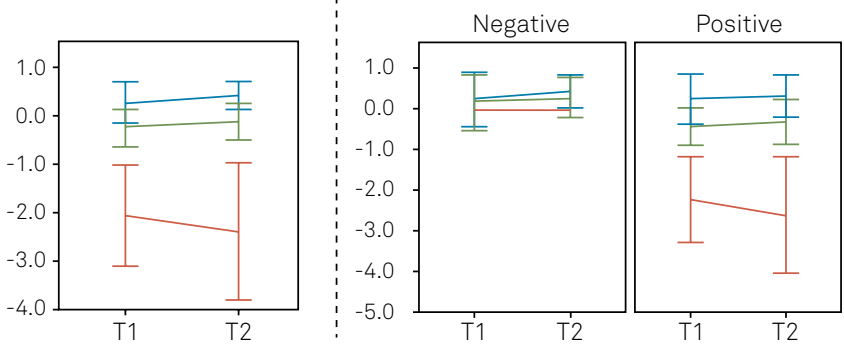

Green line: NC group; Yellow line: MCI group; Red line: AD group; T1: Baseline visit; T2: One-year follow-up.

Figure 2. Evolution line graphs for each clinical group and the respective neuropsychological test; and comparison of groups for the AD biomarker status (positive versus negative). 


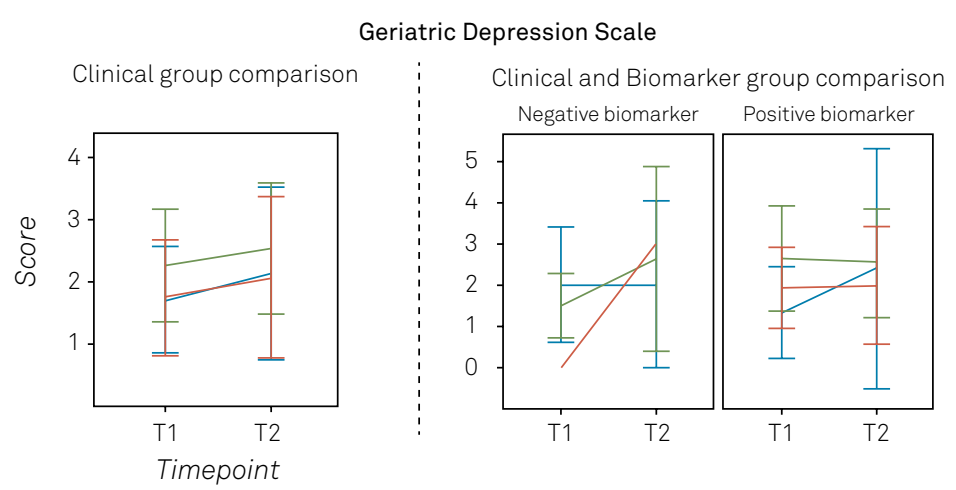

FUNCTIONAL ASSESMENT AND EVOLUTION

Functional Assessment Questionnaire

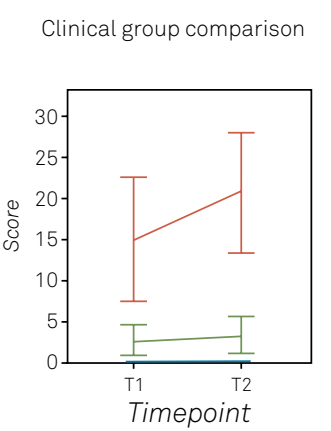

Clinical and Biomarker group comparison

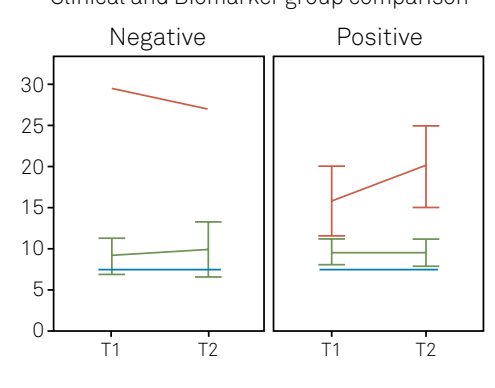

Clinical Dementia Rating (sum of bones)

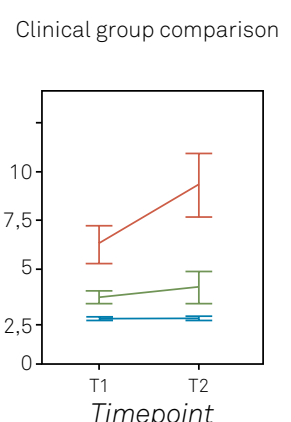

Clinical and Biomarker group comparison

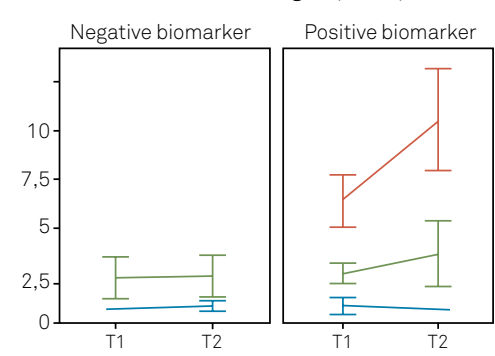

Green line: NC group; Yellow line: MCI group; Red line: AD group; T1: Baseline visit; T2: One-year follow-up.

Figure 2. Evolution line graphs for each clinical group and the respective neuropsychological test; and comparison of groups for the AD biomarker status (positive versus negative).

With regard to the short tests, the MMSE, MoCA and ADAS showed constant differences over this timespan ( $p=0.027 ; p=0.001 ; p=0.001$ respectively). However, for only the MoCA test, time had a significant impact in all three clinical groups with a significant decline of 1.35 points $(\mathrm{p}=0.018)$ in the global score.

There was no difference between groups over the timespan for the Geriatric Depression Scale. With respect to the FAQ, the AD group showed a mean decline of 18.27 points $(\mathrm{p}=0.000)$ compared with the MCI group and 20.4 points compared with the NC group ( $p=0.000)$. There was no difference between the MCI group and the NC group. The addition of an $\mathrm{AD}$ biomarker in this model showed no significant impact although it should be considered that only one participant had a negative biomarker in this group (almost all positive). The same was found for the $\mathrm{CDR}$ as the $\mathrm{AD}$ group increased by 4.34 more points compared with the mean value of the MCI and NC groups.

When analyzing the language domain, semantic fluency showed different evolutions over time between the groups ( $p=0.008$ ), insomuch as the $\mathrm{AD}$ group had a mean decline of $1.71 \mathrm{z}$ score points compared with the NC group $(\mathrm{p}=0.000)$ and $1.17 \mathrm{z}$ score points compared with the MCI group ( $\mathrm{p}=0.006$ ). For the Boston Naming Test and phonological fluency, the same significant differences found at baseline remained at the one-year visit. For these tests, the addition of $\mathrm{AD}$ biomarkers as a variable did not significantly alter the variations previously found for the established clinical group's model.

Finally, there were no significant variations over time for the non-memory tests (TMT-A and TMT-B) from those differences found at the baseline visit. Again, the addition of the biomarker variable in the model also did not change these findings.

\section{CONCLUSIONS}

In this longitudinal study, we describe variations found in neuropsychological testing of the first ADNI cohort in South America after a one-year follow-up. In this study, there was a high prevalence of positive $\mathrm{AD}$ biomarkers in the $\mathrm{AD}$ group, $92.3 \%(12 / 13)$ and a low prevalence in the $\mathrm{NC}$ group, 20\%. This means that the $\mathrm{AD}$ group represented a high probability pre-test group (the probability of having positive amyloid plaques prior to detection). The prevalence of $\mathrm{AD}$ biomarkers found in the $\mathrm{NC}$ and $\mathrm{AD}$ groups could have important considerations about the correct indication of $\mathrm{AD}$ biomarkers in the clinical setting. As previously noticed for other imaging task groups, the use of biomarkers is not recommended in these individuals due to their lack of usefulness in the diagnostic process. A different 
scenario is set for the MCI group with respect to AD biomarkers. Although MCI patients are at risk of converting to $\mathrm{AD}$, there is great uncertainty about the underlying pathology, as a non-AD pathology could be causing the clinical symptoms. Therefore, it is essential to have biomarkers that support the suspected diagnosis in investigational studies. In our cohort, almost half (48\%) of the patients diagnosed with MCI had positive amyloid detected, and the conversion rate to dementia was $20 \%$ (five patients from the MCI group). Interestingly, one of the participants who converted to dementia had negative amyloid biomarkers. Although, in this patient the possibility of a false negative result could not be ruled out ${ }^{27}$, non-AD pathology in patients with an AD-like phenotype is not uncommon ${ }^{28}$. Even though MCI patients with positive $\mathrm{AD}$ biomarkers are at higher risk of conversion to dementia, in our cohort there was no additional impact on neuropsychological tests when differentiating positive MCI versus negative MCI after one year. We acknowledge that the time period may have been too short to show significant differences, although some trends were found between groups.

In regard to the baseline analysis, it is well established that these groups represent a clinical continuum in memory impairment with different functional levels. However, not all the tests had the discriminatory power to recognize all groups, probably due to the preservation of the other cognitive domains usually found in MCI. Of note, we found that MCI patients who only had referred memory problems (amnestic type) also showed some impairment on non-memory tests, as was demonstrated by the TMT-B and semantic fluency, regardless of the expected impairments on memory tests. In regard to short tests, the MoCA test showed statistically significant differences among the three groups ( $p=0.000$ ). In contrast, the ADAS-cog could only discriminate the $\mathrm{AD}$ group from the $\mathrm{NC}$ and $\mathrm{MCI}$ groups, but not the NC group from the MCI group. This makes MoCA a suitable (and shorter) test when screening individuals with cognitive complaints and also an alternative test to the MMSE, as previously reported ${ }^{16}$.

Longitudinally, the significant differences found at baseline were similar in the one-year follow-up assessment for almost all tests, perhaps due to the relatively short time between evaluations. The exception was semantic fluency, which showed greater impairment between the $\mathrm{AD}$ group and the MCI and NC groups respectively. Impairment in semantic fluency is a characteristic feature in patients suffering from $\mathrm{AD}$, demonstrating the spread of the $\mathrm{AD}$ pathology to the entorhinal region ${ }^{29}$. In our cohort, significant differences in semantic fluency were found from the beginning in all groups, which could mean this domain is affected early. However, after one year these differences were more pronounced only in the $\mathrm{AD}$ group. In addition, the FAQ and CDR functional scales showed a pronounced worsening in the $\mathrm{AD}$ group (higher scores), leading to more significant differences compared with the NC and MCI groups. Surprisingly, the functional decline documented in the $\mathrm{AD}$ group was not accompanied by a significant worsening for the rest of the tests evaluated, except for semantic fluency.

Dividing each group and the cognitive tests according to biomarker status (positive versus negative) did not have a significant impact in our model. This was in part due to the NC group having a low prevalence of $\mathrm{AD}$ biomarkers and the $\mathrm{AD}$ group having a high prevalence, making biomarkers and the clinical diagnosis redundant. This was different for the MCI group. The ADNI model specifically focuses on patients diagnosed with MCI and aims to determine predictors of conversion to dementia. It was for this reason that more MCI participants than the other groups were included into the study. As mentioned before, half of the patients in our MCI group were positive. Therefore, we intended to demonstrate whether those "biomarker positive MCIs" would have greater impairment on neuropsychological testing, but that was not the case. We acknowledge, as limitations of the study, the limited time of the follow-up and the small cohort, which could have masked the influence of $\mathrm{AD}$ biomarkers. It is worth mentioning though, that some trends (not enough to be statistically significant) were shown between cognitive tests and biomarkers. Focusing on the MCI group, "biomarker positive MCIs" had higher mean values (worse) in the ADAS-cog. On the other hand, "negative MCIs" had shown some degree of improvement in Logical Memory, probably due to preservation of their learning skills.

\section{Acknowledgments}

Dr. Deborah Gustafson.

\section{References}

\footnotetext{
1. Brookmeyer R, Johnson E, Ziegler-Graham K, Arrighi HM. Forecasting the global burden of Alzheimer's disease. Alzheimers Dement . 2007 Jul;3(3):186-91. https://doi.org/10.1016/j.jalz.2007.04.381

2. Brookmeyer R, Gray S, Kawas C. Projections of Alzheimer 's Disease in the United States and the public health impact of delaying disease onset. 1998;88(9):1337-42. https://doi.org/10.2105/AJPH.88.9.1337

3. World Health Organization - WHO. Dementia: a public health priority. Geneva:World Health Organization; 2012.
}
4. Kalaria RN, Maestre GE, Arizaga R, Friedland RP, Galasko D, Hall K et al. Alzheimer's disease and vascular dementia in developing countries: prevalence, management, and risk factors . Lancet Neurol. 2008 Sep;7(9):812-26. https://doi.org/10.1016/S1474-4422(08)70169-8
5. Dementia C. Question- FA, Activities F. New lessons from the Alzheimer's Disease neuroimaging initiative. 2011;68(1):19-21. https://doi.org/10.1001/archneurol.2010.344


6. Russo MJ, Gustafson D, Vázquez S, Surace E, Guinjoan S, Allegri RF et al. Creation of the Argentina-Alzheimer's disease neuroimaging initiative. Alzheimer's Dement . 2014 Feb;10(1):S84-7. https://doi.org/10.1016/j.jalz.2013.09.015.

7. Hachinski V, Oveisgharan S, Romney AK, Shankle WR. Optimizing the Hachinski Ischemic Scale. Arch Neurol . 2012 Feb;69(2):169-75. https://doi.org/10.1001/archneurol.2011.16988.

8. Sheikh J, Yesavage J. Recent evidence and development of a shorter version, in clinical gerontology: a guide to assessment and intervention.d Brink T L, editor. Título do livro. New York, NY: The Haworth Press; 1986. p. 165-73.

9. Folstein MF, Folstein SE, McHugh PR. "Mini-mental state". A practical method for grading the cognitive state of patients for the clinician. J Psychiatr Res. 1975 Nov;12(3):189-98. https://doi.org/10.1016/0022-3956(75)90026-6.

10. Allegri RF, Ollari JA, Mangone CA, Arizaga RL, De Pascale A, Pellegrini M et al. El "Mini-Mental State Examination" en la Argentina: instrucciones para su administración. Rev Neurol Argent. 1999;24(1):31-5.

11. Hughes CP, Berg L, Danziger WL, Coben LA, Martin RL. A new clinical scale for the staging of dementia. Br J Psychiatry . 1982 Jun;140:566-72. https://doi.org/10.1192/bjp.140.6.566

12. Wechsler D. Wechsler memory scale. 3rd ed. San Antonio: TX Psychol; 1997.

13. McKhann G, Drachman D, Folstein M, Katzman R, Price D, Stadlan EM. Clinical diagnosis of Alzheimer's disease: report of the NINCDSADRDA Work Group under the auspices of Department of Health and Human Services Task Force on Alzheimer's Disease. Neurology. 1984 Jul;34(7):939-44. https://doi.org/10.1212/WNL.34.7.939

14. Peña-Casanova J, Aguilar M, Santacruz P, Bertran-Serra I, Hernández G, Sol JM et al. [Adaptation and normalization of the Alzheimer's disease Assessment Scale for Spain (NORMACODEM) (II)]. Neurol (Barcelona, Spain). 1997 Feb;12(2):69-77. Spanish.

15. Mangone C, De Pascale A, Gigena V, Sanguinetti R, Bauman P, Pereyra $S$ et al. Escala de evaluación para enfermedad de Alzheimer: adaptación ala lengua castellana, confiabilidad interexaminadores y en retesteo seriado. Rev Neurol Argent. 1995;20(2):31-40.

16. Nasreddine ZS, Phillips NA, Bédirian V, Charbonneau S, Whitehead V, Collin I et al. The Montreal Cognitive Assessment, MoCA: a brief screening tool for mild cognitive impairment. J Am Geriatr Soc . 2005 Apr;53(4):695-9. Https://doi.org/10.1111/j.1532-5415.2005.53221.x

17. Delgado C, Araneda A BM. Validation of the Spanish-language version of the Montreal Cognitive Assessment test in adults older than 60 years. Neurologia. 2017;:S0213-4853((17)):30102-0. htps://doi.orh/10.1016/j.nrl.2017.01.013
18. Gigena VM, Mangone CA. El test del reloj: una evaluación cognitiva rápida y sensible al deterioro incipiente. Rev Neurol Argent. 1993;18:35-42.

19. Rey. Rey Auditory Verbal Learning Test. RAVLT; L'examen Clinique En Psychologie. Paris: Presses Universitaires de France. 1964.

20. Burin D, Ramenzoni VA. Evaluación neuropsicológica del envejecimiento: normas según edad y nivel de educación. Rev Neurol Argent. 2003;28(3):149-52.

21. Serrano C, Allegri RF, Drake M, Butman J, Harris P, Nagle C et al. [A shortened form of the Spanish Boston naming test: a useful tool for the diagnosis of Alzheimer's disease]. Rev Neurol. 2001Jan;33(7):624-7. Spanish.

22. Butman J, Allegri RF, Harris PD. Fluencia verbal en español: datos normativos en Argentina. Med (Buenos Aires). 2000;60(6):561-4.

23. Tombaugh TN. Trail making Test A and B: normative data stratified by age and education. Arch Clin Neuropsychol . 2004 Mar;19(2):203-14. https://doi.org/10.1016/S0887-6177(03)00039-8

24. Arango-Lasprilla JC, Rivera D, Aguayo A, Rodríguez W, Garza MT, Saracho CP et al. Trail Making Test: normative data for the Latin American Spanish speaking adult population. Neuro Rehabilitation. 2015;37(4):639-61. https://doi.org/10.3233/NRE-151284

25. Pfeffer RI, Kurosaki TT, Harrah CH, Chance JM, Filos S. Measurement of functional activities in older adults in the community. J Gerontol. 1982 May;37(3):323-9. https://doi.org/10.1093/geronj/37.3.323

26. Surace E, Cohen G, Martinetto H. ChremMendez P, Martín E, Smyth E et al. Latin American experience with Alzheimer's disease cerebrospinal fluid biomarkers. J Am Geriatr Soc . 2013 Jul;61(7):1229-31. https://doi.org/10.1111/jgs.12352.

27. Cairns NJ, Ikonomovic MD, Benzinger T, Storandt M, Fagan AM, Shah AR et al. Absence of Pittsburgh compound B detection of cerebral amyloid beta in a patient with clinical, cognitive, and cerebrospinal fluid markers of Alzheimer disease: a case report. Arch Neurol . 2009 Dec;66(12):1557-62. https://doi.org/10.1001/archneurol.2009.279

28. Piguet O, Halliday GM, Creasey H, Broe GA, Kril JJ. Frontotemporal dementia and dementia with Lewy bodies in a case-control study of Alzheimer's disease. Int Psychogeriatr . 2009 Aug;21(4):688-95. https://doi.org/10.1017/S1041610209009454

29. Hodges JR, Patterson K. Is semantic memory consistently impaired early in the course of Alzheimer's disease? Neuroanatomical and diagnostic implications. Neuropsychologia . 1995 Apr;33(4):441-59. https://doi.org/10.1016/0028-3932(94)00127-B 